\title{
SPECTROSCOPY AND PHOTOMETRY OF THE DOUBLE-LINED BINARY HD 149420
}

\author{
Francis C. Fekel ${ }^{1}$ and Gregory W. Henry \\ Center of Excellence in Information Systems, Tennessee State University, 3500 John A. Merritt Boulevard, \\ Box 9501, Nashville, TN 37209; fekel@evans.tsuniv.edu, henry@schwab.tsuniv.edu \\ Received 2005 October 5; accepted 2005 November 10
}

\begin{abstract}
We have used new spectroscopic and photometric observations of HD 149420 to obtain an improved orbital element solution and determine some basic properties of the system. This double-lined binary has an orbital period of 3.39430 days, a circular orbit, and a mass ratio of 0.52 . The primary is somewhat evolved, with an A9 IV spectral type, while the secondary is estimated to be an F5: dwarf. The secondary may be synchronously rotating, but the primary is rotating substantially slower than its synchronous velocity. Our photometric observations confirm the light variability and period found by Hipparcos. We determine a photometric period of 1.698 days, essentially half the value of the orbital period, with a peak-to-peak amplitude of $0.028 \mathrm{mag}$ in $V$. In addition to this ellipsoidal light variation, we also detect more rapid, low-amplitude variability with a period of 0.076082 days and a peak-to-peak $V$ amplitude of $0.008 \mathrm{mag}$, as well as a possible second short 0.059256 day period with a peak-to-peak amplitude of only 0.005 mag. This indicates that the A9 IV primary in HD 149420 is a pulsating $\delta$ Scuti variable. Our binary model that best fits both the radial velocity and photometric observations indicates the possibility of extremely shallow grazing eclipses, a circumstance not unexpected given the large minimum masses from the orbital solution, the evolved nature of the primary, and the short orbital period.
\end{abstract}

Key words: binaries: spectroscopic — stars: variables: other

Online material: machine-readable table

\section{INTRODUCTION}

HD 149420 [32 Herculis, HIP 81066; $\alpha=16^{\mathrm{h}} 33^{\mathrm{m}} 29.1, \delta=$ $30^{\circ} 29^{\prime} 57^{\prime \prime}$.0 (J2000.0), $\left.V=6.87 \mathrm{mag}\right]$ was one of 97 stars identified as a spectroscopic binary by Adams et al. (1924) from observations obtained at Mount Wilson Observatory. With an additional 31 spectrograms acquired at the Dominion Astrophysical Observatory, McKellar (1935) determined a single-lined, nearly circular spectroscopic orbit with a period of 3.39430 days. This spectroscopic binary also has a visual companion about 4 " distant and 7 mag fainter (e.g., Batten et al. 1989). The visual binary is known by several names, including BU 818 AB and ADS 10116 AB. Although the spectroscopic primary was called a probable Am star by W. P. Bidelman (Abt \& Bidelman 1969), that abundance peculiarity has not been detected by others. For example, Floquet (1975) classified HD 149420 as F0 III, and Abt (1985) called it A9 IV.

The Hipparcos mission team (Perryman et al. 1997) discovered the light variability of HD 149420 and determined a period of 1.6972 days but did not hazard a guess as to its variability type. Examining the Hipparcos results, Paunzen \& Maitzen (1998) suggested that HD 149420 is a possible $\gamma$ Doradus variable (Kaye et al. 1999). Thus, in their survey of $\gamma$ Doradus candidates, Fekel et al. (2003) obtained two spectroscopic observations at red wavelengths, where they detected weak lines of the spectroscopic secondary. Fekel et al. (2003) also noted that the orbital period is twice as long as the period of light variability, and so they concluded that HD 149420 is an ellipsoidal variable rather than a $\gamma$ Doradus star.

Following up on the results of Fekel et al. (2003), we have continued spectroscopic observations of the short-period binary,

\footnotetext{
${ }^{1}$ Visiting Astronomer, Kitt Peak National Observatory, National Optical Astronomy Observatory, operated by the Association of Universities for Research in Astronomy, Inc., under cooperative agreement with the National Science Foundation.
}

components $\mathrm{Aa}$ and $\mathrm{Ab}$, to determine an improved orbit and some basic parameters of the system. We have also obtained and analyzed new photometric observations to search for possible eclipses in this short-period system.

\section{SPECTROSCOPIC OBSERVATIONS AND REDUCTIONS}

From 2000 July to 2005 June we obtained 23 high-resolution spectrograms of HD 149420 ( Table 1) with the Kitt Peak National Observatory (KPNO) coudé feed telescope, a coudé spectrograph, and a TI CCD detector. All of the spectrograms are centered in the red at $6430 \AA$, cover a wavelength range of about $80 \AA$, and have a resolution of $0.21 \AA$. Typical signal-to-noise ratios are 150-200. All but one of our spectra of HD 149420 show two sets of lines. A sample spectrum is presented in Figure 1 with several lines of components $\mathrm{Aa}$ and $\mathrm{Ab}$ identified.

We determined radial velocities with the IRAF cross-correlation program FXCOR (Fitzpatrick 1993), fitting a Gaussian function to the cross-correlation peak of each component. The IAU radial velocity standard stars $\beta$ Vir, HR 5694, and HR 7560 were used as cross-correlation reference stars. These three late-F dwarfs have radial velocities, adopted from Scarfe et al. (1990), of 4.4, 54.4, and $0.0 \mathrm{~km}^{-1}$, respectively. Lines in the wavelength region redward of $6445 \AA$ are not particularly suitable for measurement because most features are blends, and there are a number of modest-strength water vapor lines. Thus, the radial velocities were determined from lines in the region $6385-6445 \AA$. However, this $60 \AA$ portion of the spectrum is so small that a mismatch, caused by the varying strength of line blends, between the spectral type of HD 149420 and that of the cross-correlation standard can significantly alter the measured velocity. Thus, instead of crosscorrelating this entire $60 \AA$ wavelength region, only the wavelength regions around the five strongest and least blended lines, the $\mathrm{Fe}_{\mathrm{I}}$ lines at $6394,6412,6421$, and $6431 \AA$ plus the $\mathrm{Ca}$ I line at $6439 \AA$ A, were cross-correlated. 
TABLE 1

Radial Velocities of HD 149420

\begin{tabular}{|c|c|c|c|c|c|}
\hline $\begin{array}{l}\text { Heliocentric Julian Date } \\
\quad(\text { HJD }-2,400,000)\end{array}$ & Phase & $\begin{array}{c}V_{\mathrm{Aa}} \\
\left(\mathrm{km} \mathrm{s}^{-1}\right)\end{array}$ & $\begin{array}{l}(O-C)_{\mathrm{Aa}} \\
\left(\mathrm{km} \mathrm{s}^{-1}\right)\end{array}$ & $\begin{array}{c}V_{\mathrm{Ab}} \\
\left(\mathrm{km} \mathrm{s}^{-1}\right)\end{array}$ & $\begin{array}{l}(O-C)_{\mathrm{Ab}} \\
\left(\mathrm{km} \mathrm{s}^{-1}\right)\end{array}$ \\
\hline $51,736.727 \ldots$ & 0.194 & 9.3 & -0.5 & -61.6 & -2.1 \\
\hline $51,742.788 \ldots \ldots \ldots \ldots \ldots \ldots \ldots$ & 0.979 & 54.3 & 0.2 & -143.4 & 1.3 \\
\hline $52,014.949 \ldots \ldots$ & 0.161 & 21.7 & -0.7 & -82.6 & 1.2 \\
\hline $52,015.948 \ldots \ldots$ & 0.455 & -80.7 & -0.8 & 114.0 & 1.2 \\
\hline $52,016.963 \ldots \ldots$. & 0.754 & -12.8 & -0.8 & $\ldots$ & $\ldots$ \\
\hline $52,017.959 .$. & 0.048 & 50.4 & -1.2 & -142.4 & -2.5 \\
\hline $52,327.042 \ldots$ & 0.107 & 40.5 & 0.8 & -113.2 & 3.8 \\
\hline $52,328.051 \ldots \ldots \ldots \ldots \ldots \ldots \ldots$ & 0.404 & -69.9 & 0.6 & 92.5 & -2.4 \\
\hline $52,392.939 \ldots \ldots$ & 0.521 & -81.5 & 0.4 & 114.8 & -2.0 \\
\hline $52,540.621 \ldots \ldots$ & 0.030 & 54.0 & 0.5 & -142.3 & 1.2 \\
\hline $52,706.992 \ldots \ldots \ldots$ & 0.045 & 52.5 & 0.5 & -138.5 & 2.1 \\
\hline 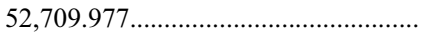 & 0.924 & 47.9 & 0.9 & -134.5 & -3.4 \\
\hline 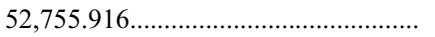 & 0.458 & -80.5 & -0.3 & 112.3 & -1.1 \\
\hline $52,757.913 \ldots \ldots$ & 0.047 & 51.2 & -0.6 & -143.2 & -3.0 \\
\hline $53,170.807 \ldots \ldots$ & 0.690 & -40.0 & -0.7 & 33.7 & -1.1 \\
\hline $53,173.791 \ldots \ldots$. & 0.569 & -76.8 & -0.6 & 106.4 & 0.6 \\
\hline 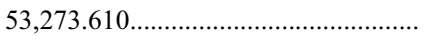 & 0.977 & 53.5 & -0.4 & -144.3 & 0.1 \\
\hline $53,275.616 \ldots \ldots \ldots$ & 0.568 & -75.8 & 0.6 & 107.1 & 0.9 \\
\hline 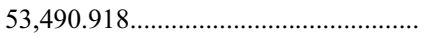 & 0.998 & 54.9 & 0.2 & -148.8 & -3.0 \\
\hline 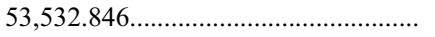 & 0.350 & -53.7 & 0.7 & 63.4 & -0.4 \\
\hline 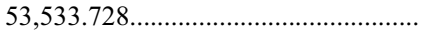 & 0.610 & -66.2 & 0.5 & 85.7 & -1.8 \\
\hline 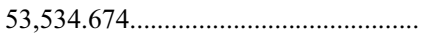 & 0.889 & 39.5 & 0.9 & -118.0 & -3.0 \\
\hline 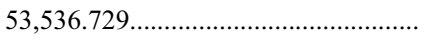 & 0.494 & -82.0 & 0.5 & 118.8 & 1.0 \\
\hline
\end{tabular}

Velocity measurement of the secondary was more complicated, since, as shown in Figure 1, its lines in the $6430 \AA$ A region are only $2 \%-3 \%$ deep. Because those lines are so weak, their velocities may be significantly affected by blends with various primary lines. Which lines of the secondary are blended depends on orbital phase and the corresponding velocity separation of the components. Thus, we first made a visual comparison of each spectrum of HD 149420 with its cross-correlation reference star to identify the least blended lines of the secondary for cross-correlation and velocity measurement. Of the lines of the secondary, typically only one or two per spectrogram provided useful velocities. The secondary velocities of the two observations obtained by Fekel et al. (2003) were remeasured, and the velocity for JD 2,451,742 was revised.

\section{PHOTOMETRIC OBSERVATIONS AND REDUCTIONS}

We obtained our photometry of HD 149420 during the 2005 observing season with the T2 $0.25 \mathrm{~m}$ automatic photoelectric telescope (APT) at Fairborn Observatory in the Patagonia Mountains

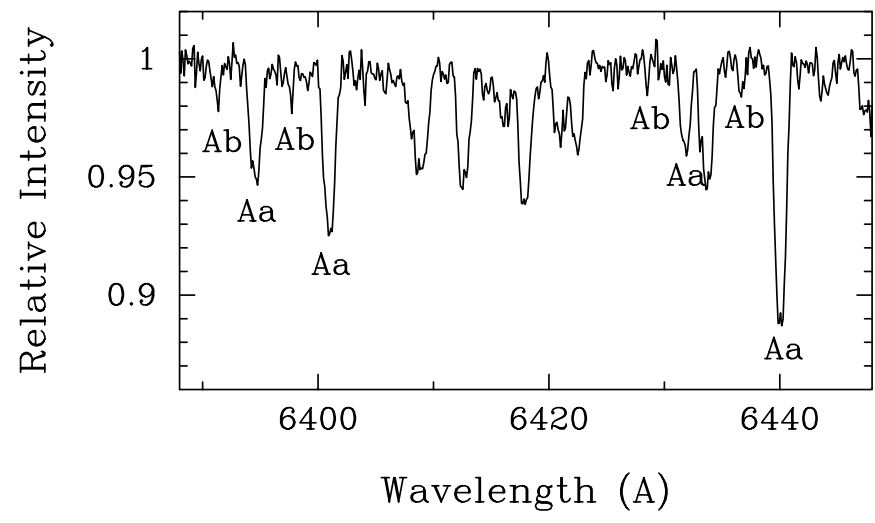

FIG. 1.-Spectrum of HD 149420 in the 6430 Å region. Several lines of components $\mathrm{Aa}$ and $\mathrm{Ab}$ are identified. of southern Arizona. This APT is equipped with a modified, temperature-stabilized SSP-3 photometer and photodiode detector from Optec, Inc., and acquires data through Johnson $V, R$, and $I$ filters. The APT is programmed to measure stars in the following sequence, termed a "group observation": $K$, sky, $C, V, C, V$, $C, V, C$, sky, $K$, where $K$ is a check star, $C$ is the comparison star, and $V$ is the program star. A total of 194 group observations of HD 149420 were obtained with the APT with HD 149044 ( $V=$ 7.17, $B-V=0.573$, F9 IV) as the comparison star and HD $151087(V=6.02, B-V=0.32$, F2 III-IV $)$ as the check star.

To create group means for each group observation, three variable-minus-comparison $(V-C)$ and two check-minuscomparison $(K-C)$ differential magnitudes in each photometric band were computed and averaged. The group means were then corrected for differential extinction with nightly extinction coefficients, transformed to the Johnson system with yearly mean transformation coefficients, and treated as single observations thereafter. The external precision of the group means, based on standard deviations for pairs of constant stars, is typically $\sim 0.006 \mathrm{mag}$ on good nights with this telescope. Group mean differential magnitudes with internal standard deviations greater than 0.02 mag were discarded. The individual differential magnitudes are given in Table 2. Further details of telescope operations and data reduction procedures can be found in Henry (1995a, 1995b).

\section{ORBIT}

Adopting the period of McKellar (1935), preliminary orbital elements for 23 velocities of the primary were computed with BISP (Wolfe et al. 1967), a computer program that implements a slightly modified version of the Wilsing-Russell method. The orbit was then refined with SB1 (Barker et al. 1967), a program that uses differential corrections. An orbit for the 22 KPNO velocities of the secondary also was computed. The variances of the solutions for the primary and secondary resulted in weights of 0.1 for the secondary velocities relative to those of the primary. Then the spectroscopic orbits of the primary and secondary were refined 
TABLE 2

Photometric Observations of HD 149420

\begin{tabular}{|c|c|c|c|c|c|c|}
\hline $\begin{array}{l}\text { Heliocentric Julian Date } \\
\quad(\text { HJD }-2,400,000)\end{array}$ & $\begin{array}{c}\text { Variable } V \\
\text { (mag) }\end{array}$ & $\begin{array}{l}\text { Variable } R \\
\text { (mag) }\end{array}$ & $\begin{array}{l}\text { Variable } I \\
\quad(\mathrm{mag})\end{array}$ & $\begin{array}{l}\text { Check } V \\
\text { (mag) }\end{array}$ & $\begin{array}{l}\text { Check } R \\
\text { (mag) }\end{array}$ & $\begin{array}{c}\text { Check } I \\
\text { (mag) }\end{array}$ \\
\hline $53,516.6809$. & -0.304 & -0.035 & 0.137 & -1.144 & -0.961 & -0.818 \\
\hline $53,516.7294 .$. & 99.999 & 99.999 & 0.147 & 99.999 & 99.999 & -0.806 \\
\hline $53,516.8881 \ldots$ & 99.999 & -0.063 & 0.136 & 99.999 & 99.999 & 99.999 \\
\hline $53,517.8281 \ldots$ & 99.999 & -0.046 & 0.136 & 99.999 & 99.999 & -0.810 \\
\hline $53,517.8851 \ldots \ldots \ldots \ldots \ldots \ldots \ldots \ldots \ldots \ldots$ & 99.999 & 99.999 & 0.138 & 99.999 & 99.999 & 99.999 \\
\hline 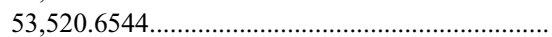 & -0.274 & -0.034 & 0.140 & -1.160 & -0.954 & -0.804 \\
\hline
\end{tabular}

Note.-Table 2 is published in its entirety in the electronic edition of the Astronomical Journal. A portion is shown here for guidance regarding its form and content.

with a modified version of SB1, which uses differential corrections to determine simultaneously the elements of the two components. Since the eccentricity of this solution is so low, $0.006 \pm 0.006$, a circular-orbit solution was computed with SB2C (D. Barlow 1998, private communication), which also uses differential corrections to determine the elements. The tests of Lucy \& Sweeney (1971) indicated that the circular-orbit solution is to be preferred, and so its elements, along with several related parameters derived from the values of those elements, are given in Table 3. For a circular orbit the element $T$, a time of periastron passage, is undefined. Thus, as recommended by Batten et al. (1989), $T_{0}$, a time of maximum velocity for the primary, is given instead.

We also computed several orbital solutions for the velocities of McKellar (1935). However, because of the uncertain zero point for those velocities, none of them have been included in our analysis.

The phases of the 23 KPNO observations and the velocity residuals to the computed curves are included in Table 1. The KPNO velocities and the computed velocity curves for components Aa and $\mathrm{Ab}$ are compared in Figure 2, where zero phase is a time of maximum velocity of the primary component.

\section{PHOTOMETRIC ANALYSIS}

The 182 Johnson $V$ variable star-minus-comparison star $(V-C)$ differential magnitudes of HD 149420 from Table 2 are plotted in Figure 3 (top). Up to several group observations were acquired by the APT each night at intervals of $2 \mathrm{hr}$. However, on two nights (JD 2,453,525 and JD 2,453,535) the APT monitored the star for several hours around the time of primary eclipse predicted by the orbital elements to search for possible shallow eclipses that might have been missed by the Hipparcos mission. The standard deviation of the observations from their mean is

TABLE 3

Orbital Elements of HD 149420

\begin{tabular}{|c|c|}
\hline Parameter & Value \\
\hline$P$ (days) & $3.3943056 \pm 0.0000091$ \\
\hline$T_{0}(\mathrm{HJD})$ & $2,452,635.5596 \pm 0.0018$ \\
\hline$\gamma\left(\mathrm{km} \mathrm{s}^{-1}\right)$ & $-13.93 \pm 0.14$ \\
\hline$K_{\mathrm{Aa}}\left(\mathrm{km} \mathrm{s}^{-1}\right) \ldots \ldots \ldots \ldots$ & $68.60 \pm 0.17$ \\
\hline$K_{\mathrm{Ab}}\left(\mathrm{km} \mathrm{s}^{-1}\right) \ldots \ldots$ & $131.85 \pm 0.53$ \\
\hline e & 0.0 adopted \\
\hline$a_{\mathrm{Aa}} \sin i(\mathrm{Gm})$ & $3.2020 \pm 0.0078$ \\
\hline$a_{\mathrm{Ab}} \sin i(\mathrm{Gm})$ & $6.1542 \pm 0.0246$ \\
\hline 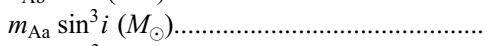 & $1.868 \pm 0.018$ \\
\hline$m_{\mathrm{Ab}} \sin ^{3} i\left(M_{\odot}\right) \ldots \ldots \ldots \ldots$ & $0.9717 \pm 0.0065$ \\
\hline \multicolumn{2}{|l|}{ Standard error of an observation } \\
\hline of unit weight $(\mathrm{km} \mathrm{s}$ & 0.7 \\
\hline
\end{tabular}

$0.012 \mathrm{mag}$, compared to the typical precision of $0.006 \mathrm{mag}$ with this telescope, indicating photometric variability in at least one of the two stars. A power spectrum of the $V-C$ observations is shown in Figure 3 (middle), computed by the method of Vanicek (1971) described in Henry et al. (2001). A similar analysis of the check star-minus-comparison star $(K-C)$ observations failed to find any significant periodicity, demonstrating that the observed variability arises in HD 149420. The best frequency is 0.5888 day $^{-1}$, corresponding to a period of $1.698 \pm 0.003$ days. This is exactly half of the radial velocity period within the uncertainties. Therefore, the observations are replotted in Figure 3 (bottom), with phases computed from the better determined orbital period and a time of maximum radial velocity of the primary star from Table 3 . The two minima occurring at phases 0.25 and 0.75 provide additional confirmation of the claim of Fekel et al. (2003) that the photometric variability results from the ellipticity effect in the binary. The peak-to-peak amplitude of the ellipticity effect is $0.028 \mathrm{mag}$ in $V$. We further analyze the ellipticity variations in $\S 8$.

An additional power spectrum of the $V-C$ observations was computed with the Vanicek (1971) method by fixing the 0.589222 day $^{-1}$ frequency that corresponds to half the spectroscopically determined orbital period. The Vanicek (1971) method differs from prewhitening for a given frequency by fixing only that frequency and not its amplitude, phase, or mean light level. A new frequency search is carried out by simultaneously fitting a single new mean brightness level and the amplitudes and phases of all frequencies introduced as fixed parameters. The result is shown in Figure 4 (top), where a high-frequency variation of $13.1438 \pm 0.0012$ day $^{-1}$ (arrow), corresponding to a period of

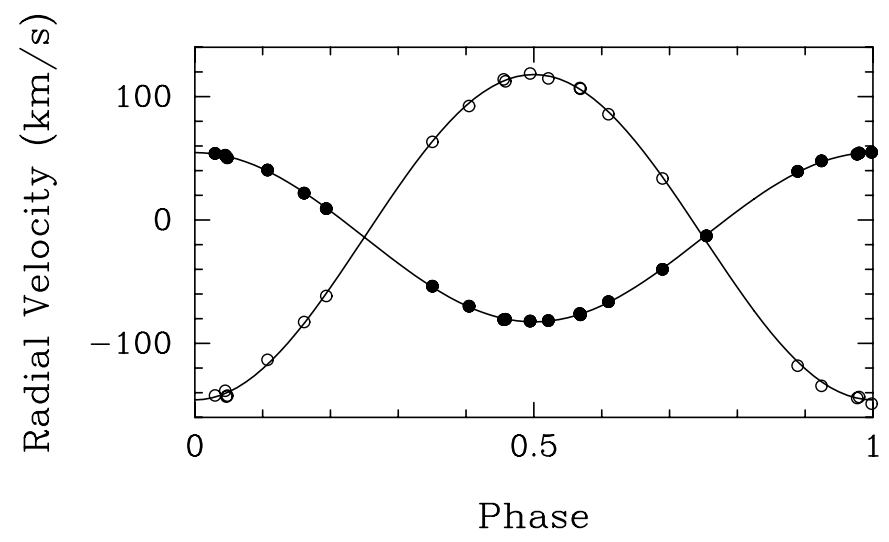

FIG. 2.-Computed radial velocity curves of HD 149420 compared with our KPNO radial velocities. Filled circles represent component Aa; open circles represent component $\mathrm{Ab}$. Zero phase is a time of maximum radial velocity of the primary. 

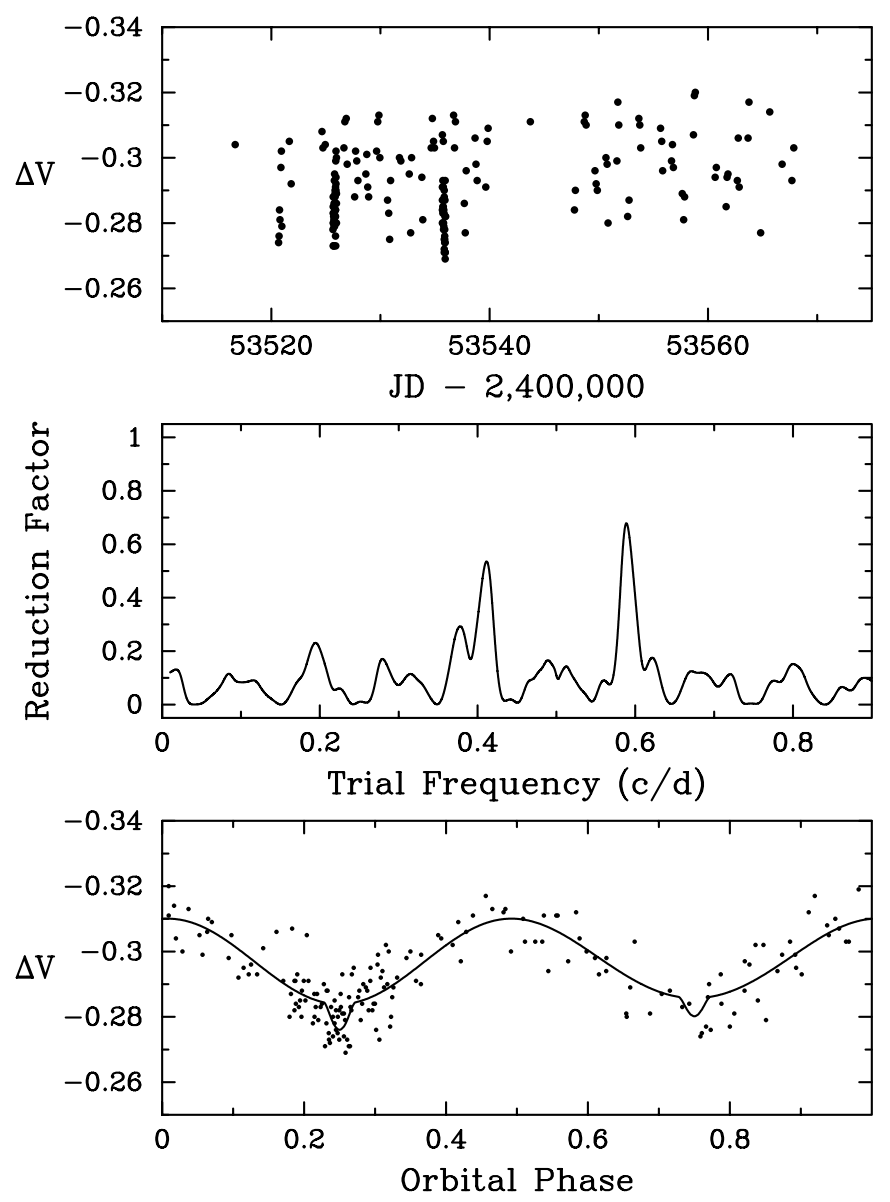

FIG. 3.-Top: Complete set of Johnson $V$ photometric observations, acquired with the T2 APT during the 2005 observing season, plotted against Julian Date. Middle: Power spectrum of the photometric observations. The best frequency is 0.5888 day $^{-1}$, corresponding to a period of $1.698 \pm 0.003$ days, which is exactly half of the observed radial velocity period within the uncertainties. Bottom: Photometric observations phased with the 3.394 day orbital period and time of maximum radial velocity from Table 3 . The solid curve represents our binary model that best fits both the radial velocity and photometric observations and indicates the possibility of extremely shallow grazing eclipses.

$0.076082 \pm 0.000007$ days, becomes apparent. This period was also fixed and the power spectrum in Figure 4 (middle) computed. The strongest peak in this power spectrum is $16.8758 \pm$ 0.0010 day $^{-1}$ (arrow), corresponding to a period of $0.059256 \pm$ 0.000004 days. This indicates a possible second high-frequency variation in HD 149420. Neither of these two high frequencies was found in the $K-C$ observations, so they are both intrinsic to HD 149420. Finally, the 16.8758 day $^{-1}$ frequency was also fixed and the power spectrum in Figure 4 (bottom) computed. No additional frequencies appear significantly above the noise. The standard deviation of the residuals from the three-period fit is $0.0059 \mathrm{mag}$, very close to the typical $0.006 \mathrm{mag}$ precision of the APT observations, confirming that no additional periods are likely to exist in the data.

The photometric observations are phased with the 0.076082 and 0.059256 day periods and a time of minimum in Figure 5 (top and bottom, respectively). The phase curve for each period has been prewhitened to remove the ellipticity effect, as well as the other short period, to improve the visibility of these lowamplitude variations. Both phase curves appear as low-amplitude sinusoids. The peak-to-peak amplitudes of the top and bottom light curves are $0.008 \pm 0.001$ and $0.005 \pm 0.001$, respectively. The primary A9 IV component in HD 149420 lies near the center

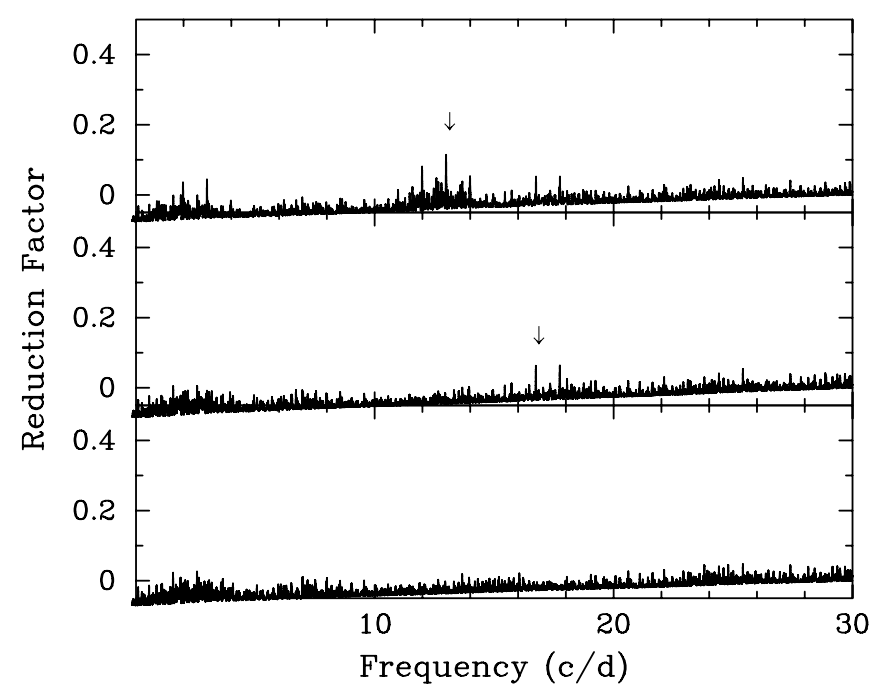

FIG. 4.-Top: Power spectrum of the Johnson $V$ photometric observations with twice the orbital frequency fixed to remove the ellipticity effect. A second higher frequency of 13.1438 day $^{-1}$ (arrow), corresponding to a short period of $0.076082 \pm$ 0.000007 days, is apparent. Middle: Power spectrum resulting from also fixing the 13.1438 day $^{-1}$ frequency. A possible second high frequency of 16.8758 day $^{-1}$ appears (arrow), corresponding to a period of 0.059256 days. Bottom: Power spectrum resulting from fixing all three frequencies. No other frequency appears significantly above the noise.

of the $\delta$ Scuti instability strip (Breger 2000), and both periods are within the $\delta$ Scuti period range (Breger 2000). Thus, in addition to being an ellipsoidal variable, it appears that this component is a low-amplitude $\delta$ Scuti pulsator. The ratio of the second to the first period is 0.779 , very close to the 0.761 period ratio of the first overtone to the fundamental pulsation mode for a typical $\delta$ Scuti star (Breger 1979). This suggests that the second, weaker period may indeed be real.

\section{SPECTRAL TYPES, MAGNITUDE DIFFERENCE, AND ROTATIONAL VELOCITIES}

W. P. Bidelman (Abt \& Bidelman 1969) called HD 149420 a probable Am star. In that same paper W. W. Morgan classified it as A9 IVsp, with the spectral peculiarity being that $\lambda 4426$ was weak. Because of Bidelman's classification, HD 149420 has appeared in various lists and surveys of Am stars. However, more recent classifications are similar to Morgan's, and no enhancement of metallic lines has been detected. Floquet (1975) classified

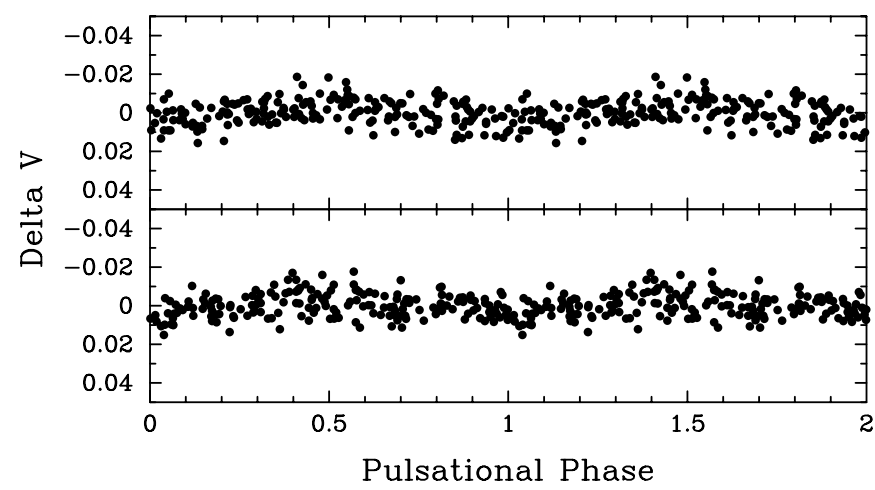

FIG. 5.- Johnson $V$ photometric observations phased with the 0.076082 (top) and 0.059256 day periods (bottom). The phase curve for each period has been prewhitened to remove the ellipticity effect, as well as the other short period. Since the primary A9 IV star in HD 149420 lies near the center of the $\delta$ Scuti instability strip, it appears that it is a low-amplitude $\delta$ Scuti pulsator, in addition to being an ellipsoidal variable. 
HD 149420 as F0 III, and Abt (1985) called it A9 IV. Fekel et al. (2003) used spectrum addition to determine a spectral class of A9 from one of our red-wavelength spectra and also estimated a $V$ magnitude difference of 2. Acquiring the spectra of additional reference stars, we have reexamined the classification of the components and their magnitude difference.

Strassmeier \& Fekel (1990) identified several luminositysensitive and temperature-sensitive line ratios in the $6430-6465 \AA$ region. Those critical line ratios and the general appearance of the spectrum were employed as spectral-type criteria. However, for stars that are hotter than about early G, the line ratios in the $6430 \AA$ region have little sensitivity to luminosity, so only the spectral class of an A or F star can be determined. The luminosity class is determined by computing the absolute visual magnitude with the Hipparcos parallax and comparing that magnitude to evolutionary tracks or a table of canonical values for giants and dwarfs.

The spectrum of HD 149420 was compared with those of slowly rotating late-A and early-F stars for the primary and midto late-F stars for the secondary. These reference stars, identified mostly from the lists of Abt \& Morrell (1995) and Fekel (1997), were observed at KPNO with the same telescope, spectrograph, and detector as our spectra of HD 149420. With a computer program developed by Huenemoerder \& Barden (1984) and Barden (1985), various combinations of reference-star spectra were rotationally broadened, shifted in radial velocity, appropriately weighted, and added together in an attempt to reproduce the spectrum of HD 149420 in the $6430 \AA$ region. The prominent features in this $80 \AA$ region are primarily iron lines, although there are also several calcium lines.

In agreement with Fekel et al. (2003) we classify the primary as A9, while for the secondary we estimate a spectral class of F5:. As noted previously, the strongest lines of the secondary in the $6430 \AA$ region are only about $2 \%$ deep (Fig. 1), making any estimate of the spectral class of the secondary more uncertain than usual, and so a colon has been appended to the classification. As discussed in $\S 7$, we adopt luminosity classes of subgiant and dwarf for the primary and secondary, respectively. From our analysis the $V$ magnitude difference is 2.6 and has an estimated uncertainty of 0.3 mag. Because the magnitude difference is rather large, its uncertainty affects the apparent magnitude of the secondary much more than that of the primary.

Fekel et al. (2003) determined $v \sin i=35 \mathrm{~km} \mathrm{~s}^{-1}$ for component Aa. Following the procedure given in Fekel (1997), for Ab we obtain a $v \sin i$ value of $18 \mathrm{~km} \mathrm{~s}^{-1}$ and estimate an uncertainty of $3 \mathrm{~km} \mathrm{~s}^{-1}$. We note that given the weakness of the lines of the secondary, our $v \sin i$ value for Ab might be too large, since blends with lines of the primary will tend to broaden the weak secondary lines.

\section{BASIC PROPERTIES}

From the Hipparcos catalog (Perryman et al. 1997) the $V$ magnitude and $B-V$ color of HD 149420 are 6.87 and 0.242 , respectively. The Hipparcos parallax of $0.00663 \pm 00^{\prime \prime} 00070$ (Perryman et al. 1997) corresponds to a distance of $150.8 \pm 16.1 \mathrm{pc}$. Although there may be a modest amount of interstellar reddening at such a distance, the color of the system is consistent with the spectral type of the primary, and so we have assumed no interstellar reddening. As a result, the parallax, the $V$ magnitude, and the adopted magnitude difference of the components were combined to obtain absolute magnitudes $M_{V}=1.1 \pm 0.2$ and $3.7 \pm 0.4 \mathrm{mag}$ for components $\mathrm{Aa}$ and $\mathrm{Ab}$, respectively. A $B-V$ color of 0.24 from Hipparcos (Perryman et al. 1997) for the A9 star and a $B-V$ of 0.43 from Johnson (1966) for the F5 dwarf were adopted and then used in conjunction with Table 3 of Flower (1996) to
TABLE 4

Fundamental Properties of HD 149420

\begin{tabular}{|c|c|c|}
\hline Parameter & Value & References \\
\hline$V$ (mag) . & 6.87 & 1 \\
\hline 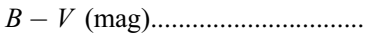 & 0.242 & 1 \\
\hline$\pi(\operatorname{arcsec}) \ldots \ldots$ & $0.00663 \pm 0.00070$ & 1 \\
\hline Spectral type of Aa.................... & A9 IV & 2 \\
\hline Spectral type of Ab.................. & F5: dwarf & 3 \\
\hline$v_{\text {Aa }} \sin i\left(\mathrm{~km} \mathrm{~s}^{-1}\right) \ldots \ldots \ldots \ldots \ldots \ldots \ldots \ldots \ldots \ldots$ & $35 \pm 2.0$ & 4 \\
\hline$v_{\mathrm{Ab}} \sin i\left(\mathrm{~km} \mathrm{~s}^{-1}\right) \ldots \ldots \ldots \ldots \ldots \ldots \ldots \ldots \ldots \ldots$ & $18 \pm 3.0$ & 3 \\
\hline 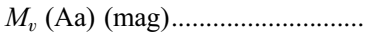 & $1.1 \pm 0.2$ & 3 \\
\hline 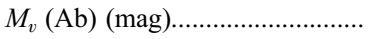 & $3.7 \pm 0.4$ & 3 \\
\hline 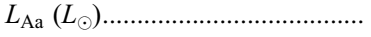 & $28.8 \pm 6.2$ & 3 \\
\hline 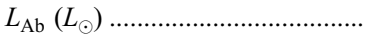 & $2.7 \pm 0.9$ & 3 \\
\hline 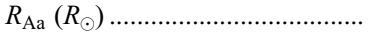 & $3.2 \pm 0.4$ & 3 \\
\hline 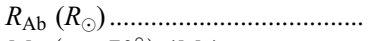 & $1.3 \pm 0.2$ & 3 \\
\hline$M_{\mathrm{Aa}}\left(i=70^{\circ}\right)\left(M_{\odot}\right) \ldots \ldots \ldots \ldots \ldots \ldots \ldots$ & 2.25 & 3 \\
\hline$M_{\mathrm{Ab}}\left(i=70^{\circ}\right)\left(M_{\odot}\right) \ldots \ldots \ldots \ldots \ldots \ldots$ & 1.17 & 3 \\
\hline
\end{tabular}

References.-(1) Perryman et al. 1997; (2) Abt 1985; (3) this work; (4) Fekel et al. 2003 .

obtain the bolometric corrections and effective temperatures of components $\mathrm{Aa}$ and $\mathrm{Ab}$. The resulting luminosities are $L_{\mathrm{Aa}}=$ $28.8 \pm 6.2$ and $L_{\mathrm{Ab}}=2.7 \pm 0.9 L_{\odot}$, while the radii are $R_{\mathrm{Aa}}=$ $3.2 \pm 0.4$ and $R_{\mathrm{Ab}}=1.3 \pm 0.2 R_{\odot}$. The uncertainties in the computed quantities are dominated by the parallax and magnitude difference uncertainties plus, to a lesser extent, the effective temperature uncertainty, which is estimated to be $\pm 200 \mathrm{~K}$. The above properties for components $\mathrm{Aa}$ and $\mathrm{Ab}$ are summarized in Table 4.

Comparison with the solar-abundance evolutionary tracks of Schaller et al. (1992) places the primary at the very end of its main-sequence evolution on a track with a mass of about $2.1 M_{\odot}$, while the secondary is close to the zero-age main sequence and has a mass of about $1.3 M_{\odot}$. From the position of the more massive star, the evolutionary tracks of Schaller et al. (1992) indicate an age of 1 billion years for the system. For the primary we adopt the A9 IV classification of Abt (1985), and the secondary we call an F5: dwarf. Comparing the mass of the primary with canonical values of Gray (1992) indicates that the primary has evolved from an early-A main-sequence star.

The masses, estimated from the evolutionary tracks, and the respective minimum masses from Table 4 produce an orbital inclination of $74^{\circ}$ for the primary and $65^{\circ}$ for the secondary. Thus, those results suggest a value of $70^{\circ}$ for the orbital inclination of the system. This mean inclination value and the minimum masses of the components (Table 3) produce masses of 2.25 and $1.17 M_{\odot}$ for $\mathrm{Aa}$ and $\mathrm{Ab}$, respectively.

\section{ECLIPSES?}

The orbital inclination also can be determined from an analysis of the ellipsoidal light curve. Thus, our Johnson $V$ photometric observations of HD 149420 were modeled with the eclipsing binary light-curve synthesis software of Bradstreet \& Steelman (2002). The orbital period and mass ratio from the radial velocity orbit were fixed, as were the various limb-darkening, gravitydarkening, and reflection coefficients appropriate to the spectral types of the stars. The stellar masses, computed in $\S 7$ with $i=70^{\circ}$, and the orbital period were used to compute the semimajor axis of the orbit with Kepler's third law; the estimated stellar radii in Table 4 were then used to derive the relative radii of the primary and secondary, 0.2233 and 0.0907 , respectively. Modeling the $V$ light curve with these parameters produced an ellipsoidal variation significantly less than that observed in the photometry. Because 
the primary star provides about $90 \%$ of the light from the system, we varied only the relative radius of the primary and the orbital inclination in a search for a solution that better reproduced the photometric variations. The photometric residuals were minimized by increasing the relative radius of the primary from 0.2233 to 0.2495 (a $12 \%$ increase but within the estimated uncertainty of the radius in Table 4) and increasing the inclination from $70^{\circ}$ to $72^{\circ}$. The resulting theoretical curve is compared with the photometric observations in Figure 3 (bottom). This best solution indicates exceedingly shallow grazing eclipses, which, given the precision of our data, may or may not be real. An ellipsoidal light-curve fit with no eclipses requires the orbital inclination to be reduced slightly to $70^{\circ}$ and a somewhat larger relative radius of 0.2560 for the primary. Clearly, the possible eclipses should be confirmed with observations of greater precision than afforded by our T2 APT. Even if eclipses do not currently take place, they will certainly occur in the not-too-distant evolutionary future, because the evolved primary will rapidly increase its radius as it moves across the Hertzsprung gap.

The radius of the primary and the orbital inclination determined from the best ellipsoidal light-curve solution are quite similar to our computed results in $\S 7$. Thus, we have not revised the properties listed in Table 4.

\section{CIRCULARIZATION AND SYNCHRONIZATION}

Matthews \& Mathieu (1992) examined 62 spectroscopic binaries with A-type primaries and periods less than 100 days. They concluded that all systems with orbital periods $\leqslant 3$ days have circular or nearly circular orbits. They also found that many binaries with periods in the range of 3-10 days have circular orbits. Thus, the circular 3.394 day orbit of HD 149420 is quite consistent with these observational results.

To determine whether the components are synchronously rotating, we combined the orbital period and our computed radii from Table 4, which results in rotational velocities of 48 and $19 \mathrm{~km} \mathrm{~s}^{-1}$ for the primary and secondary, respectively. If the orbital and rotational axes are parallel, as would be expected in such a short-period system, then our adopted inclination of $70^{\circ}$, combined with the $v \sin i$ values, results in rotational velocities of 37 and $19 \mathrm{~km} \mathrm{~s}^{-1}$ for $\mathrm{Aa}$ and $\mathrm{Ab}$, respectively. This suggests that the F5: dwarf secondary is synchronously rotating, while the A9 IV primary clearly is not. According to the evolutionary tracks of Schaller et al. (1992), the radius of the primary has approximately doubled since that star began as a zero-age mainsequence star. Given such a radius change, perhaps it is not too surprising that the primary currently has nonsynchronous rotation.

L. Boyd's dedicated efforts at Fairborn Observatory have been invaluable. We thank the referee for a careful reading of the paper. This research at Tennessee State University was supported in part by NASA grant NCC5-511 and NSF grant HRD-9706268.
Abt, H. A. 1985, ApJS, 59, 95

Abt, H. A., \& Bidelman, W. P. 1969, ApJ, 158, 1091

Abt, H. A., \& Morrell, N. I. 1995, ApJS, 99, 135

Adams, W. S., Joy, A. H., \& Sanford, R. F. 1924, PASP, 36, 137

Barden, S. C. 1985, ApJ, 295, 162

Barker, E. S., Evans, D. S., \& Laing, J. D. 1967, R. Greenwich Obs. Bull., 130, 355

Batten, A. H., Fletcher, J. M., \& MacCarthy, D. G. 1989, Publ. Dominion Astrophys. Obs., 17, 1

Bradstreet, D. H., \& Steelman, D. P. 2002, BAAS, 34, 1224

Breger, M. 1979, PASP, 91, 5

- 2000, in ASP Conf. Ser. 210, Delta Scuti and Related Stars, ed. M. Breger \& M. H. Montgomery (San Francisco: ASP), 3

Fekel, F. C. 1997, PASP, 109, 514

Fekel, F. C., Warner, P. B., \& Kaye, A. B. 2003, AJ, 125, 2196

Fitzpatrick, M. J. 1993, in ASP Conf. Ser. 52, Astronomical Data Analysis Software and Systems II, ed. R. J. Hanisch, R. V. J. Brissenden, \& J. Barnes (San Francisco: ASP), 472

Floquet, M. 1975, A\&AS, 21, 25

Flower, P. J. 1996, ApJ, 469, 355

Gray, D. F. 1992, The Observation and Analysis of Stellar Photospheres (Cambridge: Cambridge Univ. Press)

Henry, G. W. 1995a, in ASP Conf. Ser. 79, Robotic Telescopes: Current Capabilities, Present Developments, and Future Prospects for Automated Astronomy, ed. G. W. Henry \& J. A. Eaton (San Francisco: ASP), 37

\section{REFERENCES}

Henry, G. W. 1995b, in ASP Conf. Ser. 79, Robotic Telescopes: Current Capabilities, Present Developments, and Future Prospects for Automated Astronomy, ed. G. W. Henry \& J. A. Eaton (San Francisco: ASP), 44

Henry, G. W., Fekel, F. C., Kaye, A. B., \& Kaul, A. 2001, AJ, 122, 3383

Huenemoerder, D. P., \& Barden, S. C. 1984, BAAS, 16, 510

Johnson, H. L. 1966, ARA\&A, 4, 193

Kaye, A. B., Handler, G., Krisciunas, K., Poretti, E., \& Zerbi, F. M. 1999, PASP, 111,840

Lucy, L. B., \& Sweeney, M. A. 1971, AJ, 76, 544

Matthews, L. D., \& Mathieu, R. D. 1992, in IAU Colloq. 135, Complimentary Approaches to Double and Multiple Star Research, ed. H. A. McAlister \& W. I. Hartkopf (ASP Conf. Ser. 32; San Francisco: ASP), 244

McKellar, A. 1935, Publ. Dominion Astrophys. Obs., 6, 291

Paunzen, E., \& Maitzen, H. M. 1998, A\&AS, 133, 1

Perryman, M. A. C., et al. 1997, The Hipparcos and Tycho Catalogues (ESA SP-1200; Noordwijk: ESA)

Scarfe, C. D., Batten, A. H., \& Fletcher, J. M. 1990, Publ. Dominion Astrophys. Obs., 18, 21

Schaller, G., Schaerer, D., Meynet, G., \& Maeder, A. 1992, A\&AS, 96, 269

Strassmeier, K. G., \& Fekel, F. C. 1990, A\&A, 230, 389

Vaniçek, P. 1971, Ap\&SS, 12, 10

Wolfe, R. H., Jr., Horak, H. G., \& Storer, N. W. 1967, in Modern Astrophysics, ed. M. Hack (New York: Gordon \& Breach), 251 